\title{
Mycotic aneurysm of the aortic arch masquerading as systemic lupus erythematosus
}

\author{
K Chakravarty, D G I Scott
}

\begin{abstract}
The case is described of a patient with mycotic aneurysm of the aortic arch whose clinical and serological features were indistinguishable from those of systemic lupus erythematosus. Surgical resection and repair of the aneurysm resolved her clinical symptoms and the serological abnormalities.
\end{abstract}

(Ann Rheum Dis 1992; 51: 1079-1081)

The term mycotic aneurysm was introduced by Osler in 1885 to define abnormal arterial dilatation resulting from septic emboli originating from bacterial endocarditis. ${ }^{1}$ The common clinical presentations consist of pain, tenderness, and fever in association with a pulsatile mass. We describe here the case of a patient with mycotic aneurysm of the aortic arch presenting with the clinical and serological features of systemic lupus erythematosus (SLE).

\section{Case report}

A 43 year old woman presented with a two weeks' history of general malaise, flitting polyarthralgia (shoulders, elbows, knees, and wrists), and recent onset of pain and swelling of her left ankle. She also had increased hair loss from her scalp, Raynaud's phenomenon, and a photosensitive rash on her arms and legs.

In the past she had had migrainous headaches and hypertension, the latter requiring treatment with atenolol $(50 \mathrm{mg} /$ day $)$.

Physical examination revealed palmar erythema, skin rash of livedo reticularis in her legs, and synovitis with effusion in the left ankle. She \\ Department of Norfolk Norwich Hospital, Norwich NR1 3SR United Kingdom K Chakravarty D G I Scott}

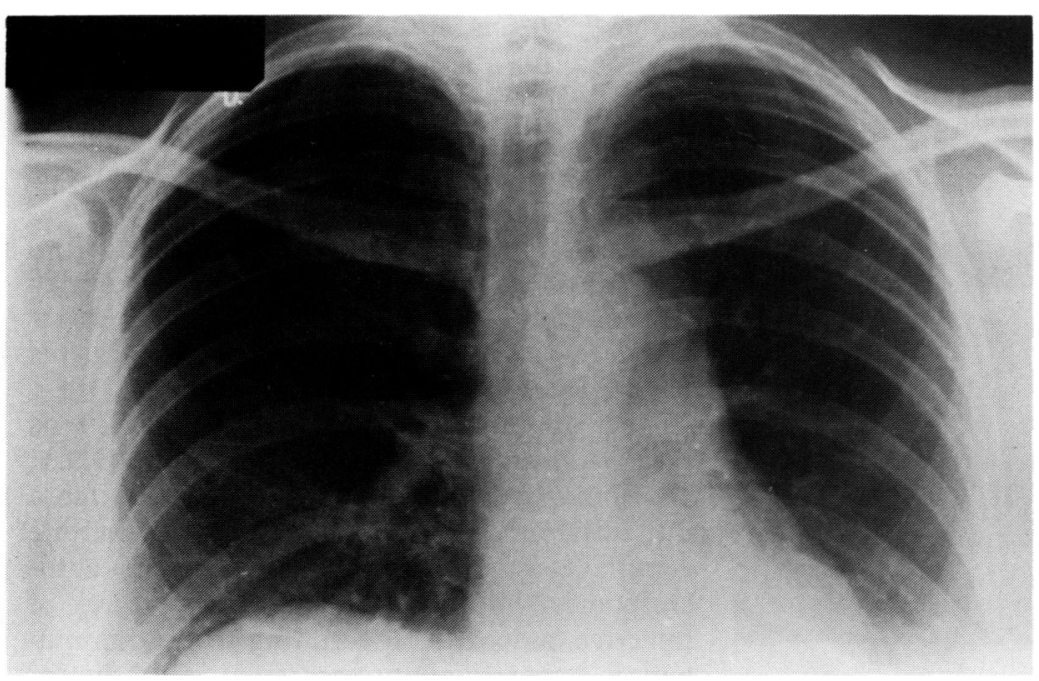

Figure 1 Chest radiograph showing a left pleural effusion. was apyrexial with a regular pulse rate of $70 /$ min, and a blood pressure of $130 / 70 \mathrm{mmHg}$ with no cardiac murmurs. General and systemic examinations were otherwise normal.

Investigations showed a haemoglobin concentration of $126 \mathrm{~g} / \mathrm{l}$, white blood cell count $12 \times 10^{9} / 1$, platelets $606 \times 10^{9} / 1$, and an erythrocyte sedimentation rate (ESR) of $124 \mathrm{~mm}$ in the first hour. Liver function tests showed increased aspartate transaminase (AST) and alanine transaminase (ALT) (AST $137 \mathrm{IU} /$, normal range <34; ALT $245 \mathrm{IU} / 1$; normal range <24), and alkaline phosphatase $(290 \mathrm{IU} / \mathrm{l}$; normal range $<120$ ). Renal functions including 24 hour urinary protein excretion and creatinine clearance were normal. Microbiological investigations including blood and urine culture were negative. Rheumatoid factor was negative but antinuclear factor was positive with increased levels of antibodies to DNA on several occasions $(87,98,76 \mathrm{IU} /$; normal range $<50)$. C reactive protein was $254 \mathrm{mg} / \mathrm{l}$ (normal range $<6 \mathrm{mg} / \mathrm{l}$ ). Antibodies to extractable nuclear antigen were not detected. Complement levels (C3 and C4) were normal but immune complex levels were increased (77 IU; normal range $<30$ ). The left ankle joint aspirate was sterile with 13 white blood cells and two red blood cells. Crystals were not seen under a polarised microscope. A dipstick test of urine detected haematuria which was subsequently confirmed by microscopic examination of urine (50 red blood cells/high power field).

A chest radiograph showed a small pleural effusion on the left (fig 1). Radiographs of the hands and feet showed soft tissue swelling of the proximal interphalangeal joints.

A provisional diagnosis of SLE was made and she was treated with ibuprofen $(400 \mathrm{mg}$ three times a day) with resolution of joint symptoms.

Four weeks later she was admitted with a one week history of an influenza-like illness, productive cough, generalised muscle ache, and polyarthralgia. She did not have chest pain, palpitations, dyspnoea, orthopnoea, nor paroxysmal nocturnal dyspnoea. On examination she appeared flushed and sweaty without pyrexia. Her pulse rate was $102 / \mathrm{min}$, regular, with no radiofemoral delay. Her blood pressure was $160 / 90 \mathrm{mmHg}$ in both arms. Physical examination was otherwise normal. Examination of her joints showed tenderness without synovitis or effusion. Laboratory investigations showed haemoglobin $112 \mathrm{~g} / 1$, white blood cell count $13.7 \times 10^{9} / 1$, ESR $94 \mathrm{~mm}$ in the first hour. AST and ALT levels were increased at 80 and $110 \mathrm{IU}$ respectively. Biochemical and microbiological investigations were otherwise normal. 
Two hours after admission she coughed up fresh blood (about $150 \mathrm{ml}$ ). A chest radiograph showed widening of the mediastinum (fig 2) and an emergency computed tomographic scan of her chest showed a large saccular aneurysm arising from the anterior aspect of the aortic arch (fig 3) with normal calibre of the ascending and descending aorta. She was then transferred to the cardiothoracic unit at Papworth Hospital where she underwent surgical resection and a graft of the aortic arch.

During the operation a large rubbery hard mass was found in the superior mediastinum extending from the mid position of the ascending aorta into the root of the neck. A portion of the left lung was also found to be adherent to the mass.

Microscopical examination of the dissected tissue showed mucoid medial degeneration with fragmentation of the elastic lamina. An active inflammation in the adventitia with haemorrhage and fibrosis was also noted. There were no specific features of giant cell reaction and no obvious vasculitis.

Microbiological culture of the dissected tissue showed exuberant growth of Haemophilus influenzae suggesting a mycotic aneurysm. She was treated with ciprofloxacin for six weeks without any detrimental sequelae.

Six months after diagnosis she remains in good health with no recurrence of her presenting

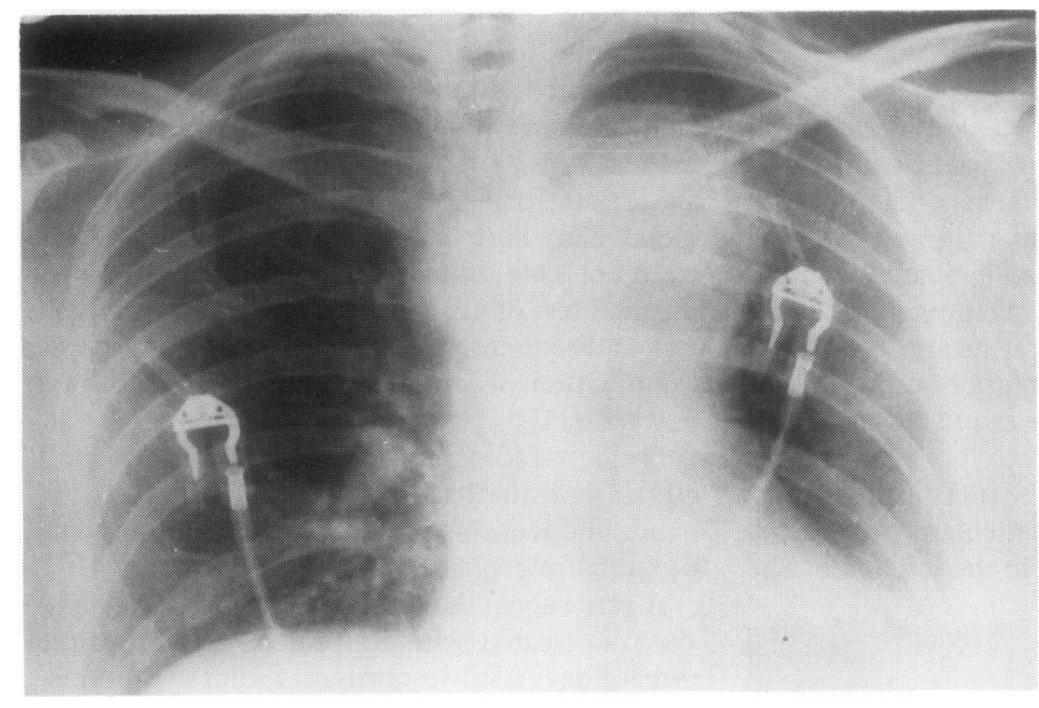

Figure 2 Chest radiograph (four weeks later) showing widening of the mediastinum.

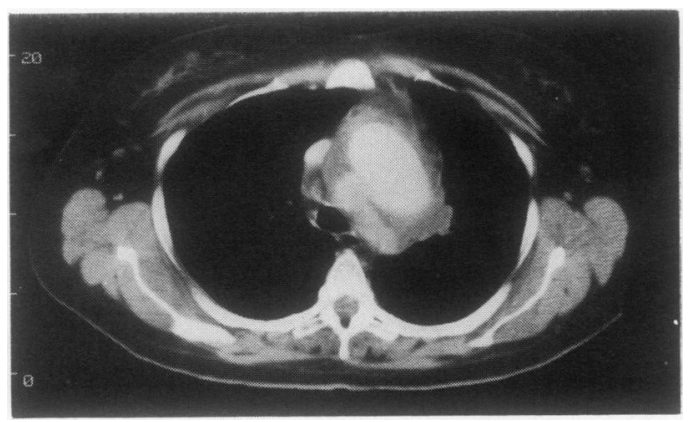

Figure 3 Computed tomographic scan of the chest showing a large aneurysm arising from the aortic arch. symptoms. Laboratory investigations showed haemoglobin $140 \mathrm{~g} / 1$, white blood cell count $6.8 \times 10^{9} / 1$ with an ESR of $18 \mathrm{~mm}$ in the first hour. Liver aminotransferase and $C$ reactive protein levels are within the normal range. Antinuclear factor and antibodies to DNA are persistently negative.

\section{Discussion}

The clinical presentation of our patient strongly suggested a diagnosis of SLE with photosensitivity, pleural effusion, non-erosive polyarthritis, antinuclear antibodies and antibodies to DNA. She thus fulfilled five of the 11 American Rheumatism Association criteria for the diagnosis of SLE. ${ }^{2}$ In addition she clearly developed a mycotic aneurysm and it was originally thought that these two diagnoses might be independent of each other. The possibility that her 'SLE' was a direct consequence of the mycotic aneurysm was suggested by the complete resolution of her clinical, biochemical, and serological abnormalities following an operation.

The high levels of $C$ reactive protein are not a usual feature of $\mathrm{SLE}^{3}$ and this, together with increased levels of transaminase suggested associated infection. Repeated blood cultures and antibodies to common viral antigens were negative but in retrospect the biochemical abnormalities probably reflected an ongoing infective process due to the mycotic aneurysm proved only by the operation.

Mycotic aneurysms usually present with pain, tenderness, and fever associated with a pulsatile mass, which may be palpable when they are located in the extremities. One study ${ }^{4}$ has described systemic symptoms such as fever, weight loss, a high ESR, and abnormal liver function tests in patients with idiopathic aortic dissection, but there has been no report of mycotic aneurysm presenting with features of a connective tissue disease such as SLE.

Mycotic aneurysms are uncommon arterial lesions associated with high mortality and morbidity from haemorrhage and sepsis. The most common organisms implicated in mycotic aortic aneurysms include salmonella in $30 \%$, staphylococcus and enterobacter, ${ }^{6}$ and pseudomonas. ${ }^{7}$ Haemophilus influenzae has not been recorded to cause mycotic aneurysm of the aortic arch in these large series. ${ }^{5-7}$ It is also usual to grow the organism in blood culture, though several cultures were negative in our patient.

The source of the infection is often $(80 \%)$ from infected heart valves, though rarely it may be the result of microbial arteritis. ${ }^{89}$ The latter seems to be the most likely explanation in this patient (an echocardiogram was normal).

The immunological abnormalities such as positive antinuclear factor, antibodies to DNA, and increased immune complexes are more difficult to explain. The presence of immune complexes, complement activation, and autoantibodies, however, are common features of infective endocarditis, resulting from immunological hyperreactivity associated with diminished function and activity of $T$ lymphocytes 
together with exaggerated B lymphocyte antibody response, due to a persistent bacterial antigenic stimulus. It is possible that a similar autoimmune mechanism may have been responsible for the immunological abnormalities seen in our patient. Certainly removal of the source of infection by an operation resulted in the complete disappearance of these serological abnormalities.

We have presented the case of a patient who apparently developed clinical and serological features of SLE associated with a mycotic aortic aneurysm due to an unusual pathogen in the absence of infective endocarditis. The clinical course of her illness strongly suggests that the infected aneurysm was responsible for her apparent lupus. Although serositis has been described as one of the major causes of high levels of $C$ reactive protein in patients with established SLE ${ }^{10}$ systemic infection including mycotic aneurysm should seriously be considered in cases of suspected SLE with high levels of $\mathrm{C}$ reactive protein.
We are grateful to Mr Frank Wells for the surgical management of this patient, and Mrs D Novis for secretarial assistance.

1 Osler W. Gulstonian lectures on malignant endocarditis. $B M \mathcal{7} 1885$; i: 467-70.

2 Tan E M, Cohen A S, Fries J F, et al. Revised criteria for the classification of systemic lupus erythematosus. Arthritis Rheum 1982; 25: 1271-7.

3 Pereira da Silva J A, Elkon K B, Hughes G R V, et al. C-reactive protein levels in systemic lupus erythematosus. A classification criterion. Arthritis Rheum 1980; 23: 770-1.

4 Turner N, Pusey C. Aortic dissection masquerading as systemic disease-the post dissection syndrome. $Q \mathcal{F}$ Med

5 Finsetti F, Abbot W M. One stage operative therapy for salmonella mycotic abdominal aortic aneurysm. Ann Surg 1974; 179: 8-11.

6 Fromm S H, Lucas C E. Obturator by-pass for mycotic aneurysm in the drug addict. Arch Surg 1970; 100: 82-3.

7 Huebl K C, Read R C. Aneurysmal abscess. Minn Med 1966 49: $11-6$.

8 Brown S L, Busuthil R W, Baker J D, et al. Bacteriologic and surgical determinants of survival in patients with mycotic surgical determinants of survival in patient

$9 \mathrm{Oz} \mathrm{M} \mathrm{C,} \mathrm{Brener} \mathrm{B} \mathrm{J,} \mathrm{Buda} \mathrm{J} \mathrm{A,} \mathrm{et} \mathrm{al.} \mathrm{A} \mathrm{ten} \mathrm{year} \mathrm{experience}$ with bacterial aortitis. F Vasc Surg 1989; 10: 439-49.

10 Ter Borg E J, Horst G, Limburg P C, et al. C-reactive protein levels during disease exaccerbations and infections in systemic lupus erythematosus. A prospective longitudinal study. F Rheumatol 1990; 17: 1642-8. 